\title{
Small-Angle X-Ray Scattering from Bulk Block Polymers in Disordered State. Estimation of $\chi$-Values from Accidental Thermal Fluctuations ${ }^{\dagger}$
}

\author{
Keiji MoRI, Hirokazu Hasegawa, and Takeji Hashimoto \\ Department of Polymer Chemistry, Faculty of Engineering, \\ Kyoto University, Kyoto 606, Japan
}

(Received December 25, 1984)

\begin{abstract}
Small-angle X-ray scattering (SAXS) from accidental thermal concentration fluctuations of block polymers in bulk and in the disordered state was studied for a polystyrenepolyisoprene diblock polymer as a function of temperature. It was shown that the experimental SAXS profiles are predictable by Leibler's theory based on the random phase approximation. Temperature dependence of $\chi$-parameter between polystyrene and polyisoprene was estimated by fitting the experimental and theoretical SAXS profiles. An effect of glass transition on the thermal concentration fluctuations was also predicted.
\end{abstract}

KEY WORDS SAXS / Block Polymers / Disordered State / Order-Disorder Transition / Random Phase Approximation / $\chi$-Parameter / Microphase Separation / Glass Transition Temperature / Accidental Thermal Fluctuations /

Nature of order-disorder transition of block polymers has recently been explored quite extensively. $^{3-8}$ In the ordered state block polymers have morphologies of their microdomains spatially arranged in periodic manners with long-range order as a consequence of the liquid-liquid microphase separation, while in the disordered state the microdomains are dissolved and the block chains are mixed with each other on the molecular level.

In this paper we shall investigate accidental thermal concentration fluctuations of block polymers in bulk and in the disordered state by means of small-angle X-ray scattering (SAXS). The experimental SAXS profiles were found to show a broad scattering maximum with its peak position independent of temperature but the peak intensity decreases with increasing temperature. $^{4,8}$ These tendencies turned out to be predictable ${ }^{5-8}$ by the theory of Leibler ${ }^{3}$ on the accidental thermal concentration fluctuations of block polymers in bulk and in the disordered state which was developed on the basis of the random phase approximation (RPA). ${ }^{9}$ The SAXS studies on a polystyrene-polyisoprene (SI) diblock polymer yielded quantitative data on the temperature dependence of the interaction parameter $\chi$ between polystyrene (PS) and polyisoprene (PI).

\section{Order-Disorder Transition of Block Polymers}

Figure 1 illustrates schematically the orderdisorder transition in block polymers consisting of $\mathrm{A}$ and $\mathrm{B}$ block chains. In the ordered state, the energetics originating from the repulsive interaction between $\mathrm{A}$ and $\mathrm{B}$ block chains outweighs the entropy originating from a random placement of the centers of the molecules, resulting in segregation of $\mathrm{A}(\mathrm{B})$

${ }^{\dagger}$ A part of this work was presented at Annual Symposiums of the Society of Polymer Science, Japan, October, $1983^{1}$ and September $1984 .^{2}$ 


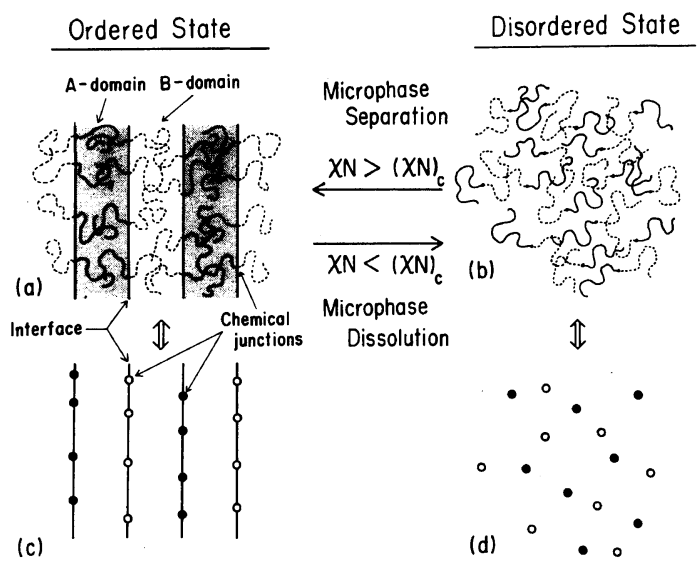

Figure 1. Concept of the order-disorder transition in block polymer system.

block chains into A (B) microdomains (Figure 1(a)). On the other hand, in the disordered state the entropy outweighs the energetics, resulting in molecular mixing of $\mathrm{A}$ and $\mathrm{B}$ chains (Figure 1(b)). This is a phenomenon common to all interacting physical systems. ${ }^{10}$ The block polymers also exhibit the orderdisorder transitions as a cooperative phenomenon. The relevant order parameter here in the block polymers is spatial segmental concentration fluctuations from the average values.

The segregation of A and B block chains into the respective microdomains in the ordered state results in a regular periodic microdomain morphology with long-range order as schematically shown in Figure 1(a) for alternating lamellar microdomains. The characteristics of the ordered state is symbolically depicted in Figure 1(c) where the chemical junctions between $\mathrm{A}$ and $\mathrm{B}$ block chains represented by the solid and open circles are confined in the narrow interfacial region (drawn by a series of vertical straight lines) with the characteristics interfacial thickness of about $20 \AA$ for SI block polymers. ${ }^{11-15}$ Generally the average end-to-end vectors are considered to orient normal to the interface. The solid and open circles then differentiate the orientations of the end-to-end vectors of the block chains, e.g., the solid (open) circles represent the junctions with their end-to-end vectors of A block chains oriented right (left). Thus the block polymers in the ordered state have the liquidcrystalline characteristics, although the molecules constituting the mesophase are the flexible chains without any mesogenic groups. On the other hand, in the disordered state the chemical junctions are randomly distributed in space and the orientations of the end-to-end vectors for $\mathrm{A}$ and $\mathrm{B}$ block chains are also random, as schematically illustrated in Figure 1(d) by a random placement of the solid and open circles. It should be noted that the systems which exhibit the spherical and cylindrical microdomains in the super-lattices in the ordered state have almost the same characteristics as described above for the alternating lamellar microdomains.

As in the case of polymer/polymer binary mixtures, ${ }^{16}$ the order-disorder transition of block polymers in bulk is characterized by a product of the total degree of polymerization $N$ and the thermodynamic interaction parameter $\chi$ as well as the fraction of one component $f_{\mathrm{K}}$ in the binary system.

$$
N=N_{\mathrm{A}}+N_{\mathrm{B}}
$$

where $N_{\mathrm{K}}(\mathrm{K}=\mathrm{A}, \mathrm{B})$ is the degree of polymerization of $\mathrm{K}$ block chain. In case of a block polymer, $f_{\mathrm{K}}$ is given by

$$
f_{\mathrm{K}}=N_{\mathrm{K}} /\left(N_{\mathrm{A}}+N_{\mathrm{B}}\right) \quad(\mathrm{K}=\mathrm{A}, \mathrm{B})
$$

In case of polymer/polymer mixtures, $f_{\mathrm{K}}$ corresponds to the volume fraction of $\mathrm{K}$ polymer with the degree of polymerization $N_{\mathrm{K}}$. The order-disorder transition points as defined by $(\chi N)_{\mathrm{c}}$ for block-polymers ${ }^{3}$ and polymer/ polymer mixtures ${ }^{16}$ are given by

$$
(\chi N)_{\mathrm{c}}=\left\{\begin{array}{cl}
10.5 & \left(\text { block polymers, } N=N_{\mathrm{A}}+N_{\mathrm{B}}\right) \\
2 & \left(\text { polymer/polymer mixtures, } N=N_{\mathrm{A}}=N_{\mathrm{B}}\right)
\end{array}\right.
$$


for $f_{\mathrm{K}}=1 / 2$ if two polymers are symmetrical with respect to their segmental sizes.

The connectivity between $\mathrm{A}$ and $\mathrm{B}$ chains by a covalent bond strongly affects $(\chi N)_{\mathrm{c}}$ and strongly enhances the miscibility of A and B chains, despite of the difference in the definitions of $\chi N$ between block polymers and polymer/polymer mixtures.

In our previous paper ${ }^{7}$ the order-disorder transition was characterized in two ways. The SAXS profiles of block polymers have at least one scattering maximum regardless of the states of the block polymers. From the peak position $\left(q_{\max }\right)$ one can estimate the apparent Bragg spacing $D$ by using Bragg's law,

$$
q_{\max }=2 \pi / D
$$

where $q$ is the scattering vector defined by

$$
q=(4 \pi / \lambda) \sin \theta
$$

$\lambda$ and $\theta$ are the wavelength of X-ray and a half of the scattering angle, respectively. The spacing $D$ in the ordered state has a definite physical meaning because the microdomains are in the super-lattices with long-range order such as one-dimensional, two-dimensional and cubic lattices for lamellar, cylindrical and spherical microdomain morphologies, respectively.

The physical meaning of spacing $D$ in the disordered state is more complicated and intuitively less clear compared with that in the ordered state. It corresponds to the wavelength of a particular Fourier component of the accidental thermal concentration fluctuations of $\mathrm{A}$ and $\mathrm{B}$ segments which gives rise to the maximum amplitude of the fluctuations. The existence of $D$ and therefore the scattering maximum is again a consequence of the connectivity of $\mathrm{A}$ and $\mathrm{B}$ chains, i.e., a phenomenon unique to block polymers. From this intuition it may be obvious that the spacing $D$ depends on $f_{\mathrm{K}}$ and the radius of gyration of the block polymer. This intuition has nicely been verified by a series of theoretical works which describe the accidental fluctuations based on the random phase approximation ${ }^{2,3,9,17,18}$ or based on Ornstein-Zernike theory. ${ }^{19}$

The cross-over behavior of $D$ in the orderdisorder transition can be summarized as ${ }^{7}$

$$
\begin{array}{ll}
D \sim N^{2 / 3}\left(\phi_{\mathrm{p}} / T\right)^{1 / 3} & \text { (ordered state) } \\
D \sim N^{1 / 2}\left(\phi_{\mathrm{p}} / T\right)^{0} & \text { (disordered state) }
\end{array}
$$

which can be used to characterize the orderdisorder transition.

An alternative way to characterize the order-disorder transition involves the intensity measurements at $q$ near $q_{\max }$ as a function of temperature. The intensity depends on $\chi N$, and if $\chi$ is written as

$$
\chi=A+B / T
$$

where $A$ and $B$ are constant values, then Leibler's theory predicts that in the disordered state

$$
\begin{aligned}
& I(q)^{-1}=F(u) / N-2 A-2 B / T \\
& \quad \text { (disordered state) }
\end{aligned}
$$

where $F(u)$ is given by eq IV-6 of ref 3 and is independent of temperature $T$. Therefore $I^{-1}$ should linearly decrease with $T^{-1}$ in the disordered state, the deviation from which is attributed to the onset of the microphase separation. In our previous papers ${ }^{5-8}$ the order-disorder transition was doubly checked with the cross-over behavior of $D$ (eq 7 and 8 ) and the intensity change with $T$ (eq 10).

\section{Scattering from the Accidental Fluctuations in the Disordered State}

The block polymer sample employed for the SAXS studies in the disordered state is an SI diblock polymer (designated hereafter as HK17) prepared by the sequential living anionic polymerization in THF (tetrahydrofuran) at $-78^{\circ} \mathrm{C}$ with $\mathrm{sec}$-butyllithium as initiator. It has the number average molecular weight $\left(M_{n}\right)$ of $8.5 \times 10^{3}$ as measured by vapor pressure osmometry and contains $50 \mathrm{wt} \%$ PS as measured by elemental analysis. The heterogeneity index of the molecular weight $\left(M_{w} / M_{n}\right)$ is 1.25 


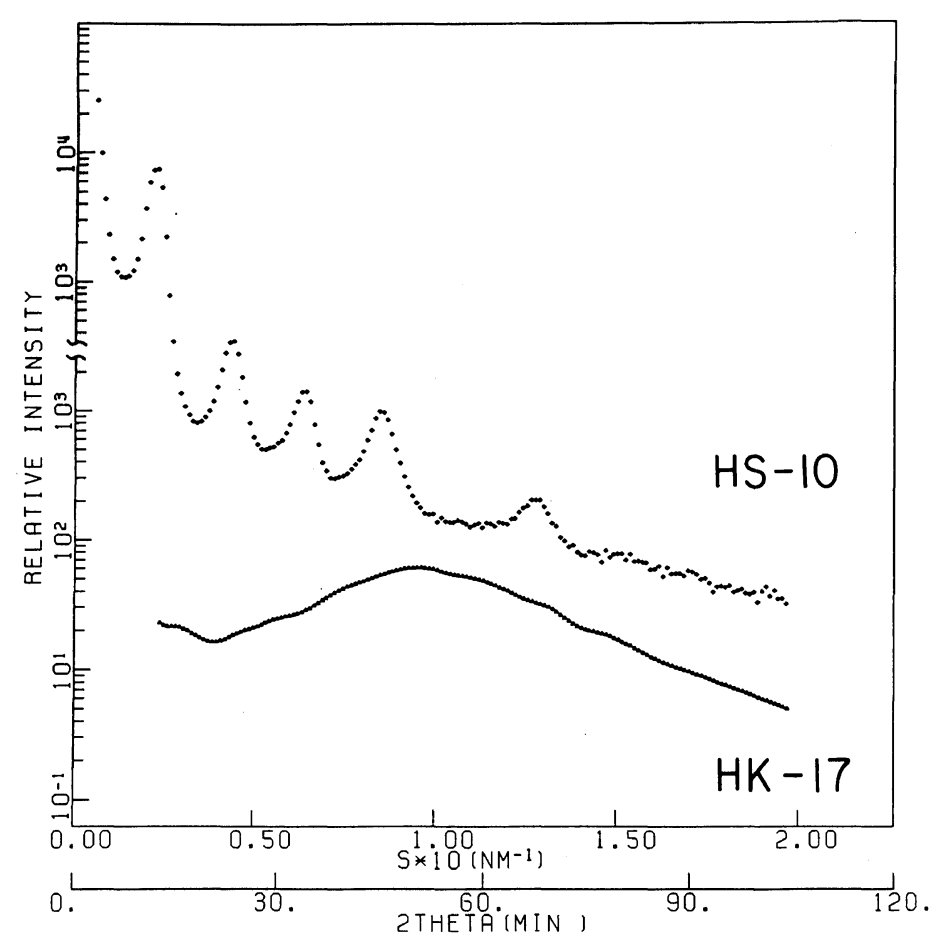

Figure 2. Comparison of the SAXS profiles of HS-10 (ordered state) and HK-17 (disordered state).

from GPC measurements $\left(M_{w}\right.$ being the weight average molecular weight). The polyisoprene block chains of SI diblock polymers synthesized in the same manner have the microstructures of 1,4-, 1,2-, and 3,4-linkages by 3,38 , and $59 \%$, respectively. ${ }^{20}$

In order to compare the scattering from the disordered state with that from the ordered state, an SI diblock polymer (designated hereafter as HS-10) was also prepared by the same technique. It has $M_{n}=8.14 \times 10^{4}$ and $M_{w} / M_{n}=1.13$, and contains $63 \mathrm{wt} \%$ of PS. The sample HS-10 has the molecular weight high enough to be in the ordered state at room temperature.

The SAXS profiles were taken with an apparatus described in detail elsewhere ${ }^{21,22}$ which consists of a $12 \mathrm{~kW}$ rotating-anode Xray generator, a graphite monochromator, a $1.5 \mathrm{~m}$ camera and a one-dimensional position sensitive proportional counter. $\mathrm{Cu} K_{\alpha}$ line $(\lambda=$ $1.54 \AA$ ) was used. The SAXS profiles for HK-
17 were corrected for absorption, air scattering, background scattering arising from the thermal diffuse scattering (TDS), and slitheight and slit-width smearings. The SAXS profiles for HS-10 were measured along the direction perpendicular to the film surface by irradiating the incident beam with a line image parallel to the film surface. The sample HS-10 has an alternating lamellar morphology with the lamellar interfaces highly oriented parallel to the film surface as already described in detail elsewhere. ${ }^{11}$ Since the line image was set parallel to the film surfaces, the profiles measured perpendicular to the film surface are free from the slit-height smearing but slightly affected by the slit-width smearing. The profiles were again corrected for absorption, air scattering and background scattering.

Figure 2 shows the corrected SAXS profiles of the cast films of HK-17 and HS-10 from toluene solutions where $s$ is defined as 


$$
s=(2 \sin \theta) / \lambda
$$

Although the SAXS profiles from the as-cast films are shown in a relative intensity scale, the relative intensity of $\mathrm{HK}-17$ and $\mathrm{HS}-10$ can be compared. In order to facilitate to show the overall profiles, a logarithmic intensity scale was chosen. HS-10 shows multiple-order scattering maxima from the single spacing $D$ (=452 $)$

$$
2 D \sin \theta=n \lambda
$$

or

$$
s D=n \quad(n, \text { integers })
$$

which is typical to an alternating lamellar microdomain morphology. On the other hand, HK-17 shows only the first order scattering maximum which is very broad and very weak compared with that of HS-10. The maximum intensity of $\mathrm{HK}-17$ is two to three order of

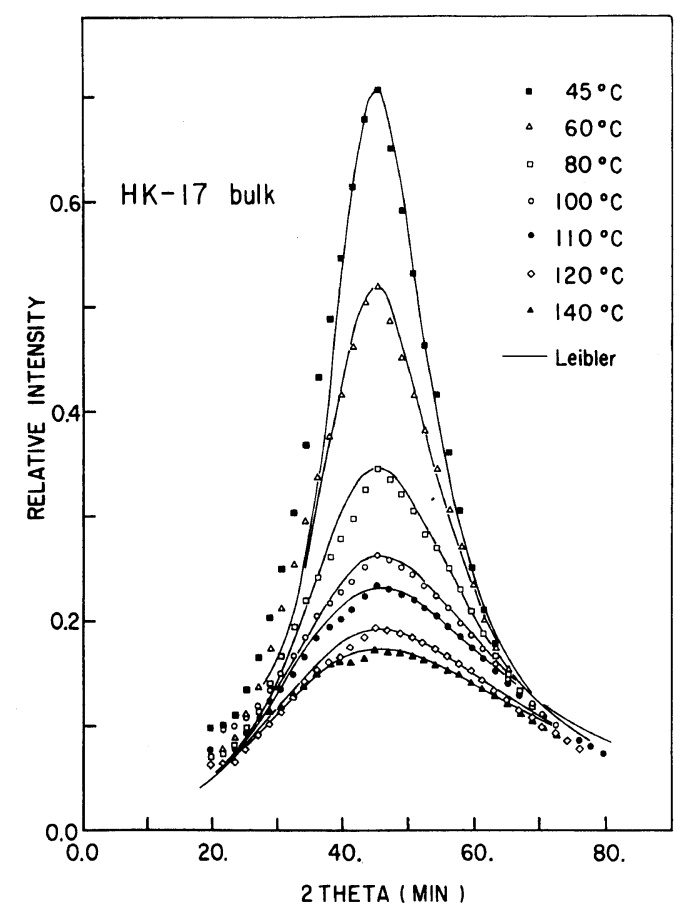

Figure 3. SAXS profiles of HK-17 in the disordered state obtaind at various temperatures and plotted in a linear scale as a function of the scattering angle. magnitudes smaller than that of HS-10. Following analyses will prove that the scattering from $\mathrm{HK}-17$ is typical to the scattering from the disordered state.

Figure 3 shows SAXS profiles from HK-17 at various temperatures where the intensity was plotted in linear scale as a function of the scattering angle $2 \theta$. The experimental profiles were shown by the discrete points, while the best-fitted theoretical profiles were shown by the solid lines. The theoretical analyses will be described in the following section.

It is clearly seen that the intensity decreases with increasing temperature but the scattering angle $2 \theta_{\max }$ at which the intensity becomes maximum is independent of temperature. The Bragg spacing $D$ as estimated from $2 \theta_{\max }$ was plotted in Figure 4 as a function of temperature.

Theoretical Analyses and Estimation of $\chi$ Parameter

The experimental evidence that the spacing is independent of temperature strongly suggests that the scattering arises from the accidental thermal fluctuations in the disordered

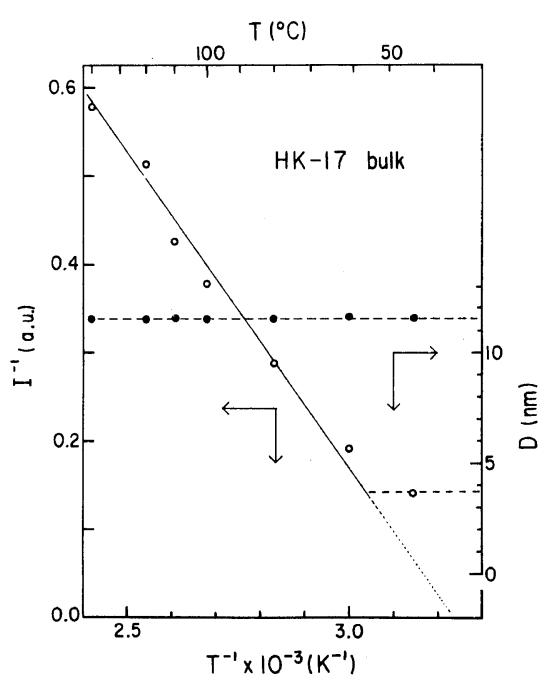

Figure 4. Reciprocal SAXS intensity (open circles) measured at $q=0.0539 \AA^{-1}$ and Bragg spacings (solid circles) at the scttering maxima of $\mathrm{HK}-17$ in bulk plotted as functions of reciprocal temperature. 
state (see eq 7 and 8 ). ${ }^{7}$ Consequently we analyzed our data in terms of Leibler's theory ${ }^{3}$ for the scattering from the disordered state. According to Leibler, the scattered intensity $S(q)$ is given by

$$
\begin{aligned}
& S(q) \sim N /(F(u)-2 \chi N) \\
& u=q^{2} R_{\mathrm{g}}^{2}
\end{aligned}
$$

where $R_{\mathrm{g}}$ is the radius of gyration of the block polymer chains in the unperturbed state.

The theoretical scattering profiles were calculated and compared with the experimental profiles as follows. Leibler's theory was originally developed for the symmetrical diblock polymers, i.e., two constituent polymers have equal segmental lengths and equal segmental densities but different contrast factors for scattering. In order to apply this theory to the SI diblock polymer, however, it needs some modification which accounts for the asymmetry, i.e., the differences in the segmental lengths and the segmental densities. Here we employed Helfand's method ${ }^{15}$ of treating the asymmetry. According to Helfand, the average segmental density $\rho_{0}$ and the average statistical segmental length $b$ for an SI diblock polymer are given by

$$
\begin{gathered}
\rho_{0}=\left(\rho_{\mathrm{OS}} \rho_{\mathrm{OI}}\right)^{1 / 2} \\
\rho_{0} b^{2}=(1 / 2) \rho_{0 \mathrm{~S}} b_{\mathrm{S}}^{2}+(1 / 2) \rho_{\mathrm{OI}} b_{\mathrm{I}}^{2}
\end{gathered}
$$

where $\rho_{0 \mathrm{~K}}(K=\mathrm{S}, \mathrm{I} ; \mathrm{S}$ and I refer to PS and PI block chains, respectively) is the number density of $\mathrm{K}$ monomer units and $b_{\mathrm{K}}$ is Kuhn's statistical segmental length for $\mathrm{K}$ polymer chains. The total degree of polymerization for the SI diblock polymer, $N$ and the $\chi$-values are defined also after Helfand by

$$
\begin{gathered}
N=\rho_{0}\left(\mathrm{~N}_{\mathrm{S}} / \rho_{0 \mathrm{~S}}+N_{\mathrm{l}} / \rho_{0 \mathrm{I}}\right) \\
\chi=\left(\delta_{\mathrm{S}}-\delta_{\mathrm{I}}\right)^{2} / \rho_{0} k_{\mathrm{B}} T
\end{gathered}
$$

where $N_{\mathrm{K}}$ is the degree of polymerization, $\delta_{\mathrm{K}}$ is the solubility parameter for $\mathrm{K}$ polymer chain and $k_{\mathrm{B}}$ is the Boltzmann constant. We defined $f_{\mathrm{K}}$ as

$$
f_{\mathrm{K}}=\rho_{0} N_{\mathrm{K}} / \rho_{0 \mathrm{~K}} N
$$

The theoretical scattering intensity was calculated as a function of the reduced scattering angle $q R_{\mathrm{g}}$ with eq 13 , and was fitted to the experimental scattering profiles. The best fit would yield the parameters $\chi \mathrm{N}$ and $R_{\mathrm{g}}$. For a given molar composition $f_{\mathrm{K}}$, the parameter $R_{\mathrm{g}}$ affects the peak position of the profiles and the parameter $\chi N$ affects the peak height.

The best fits between the theoretical and experimental curves were shown in Figure 3. A very good agreement between the theoretical and the experimental curves was obtained, suggesting that the scattering arises from the accidental thermal fluctuations in the disordered state. The $\chi N$ values which give the best fits yield the estimated values of $\chi$. The $\chi$ values on monomer units thus estimated were summarized in Table I. The $\chi$-value decreases with increasing temperature as shown in Figure 5.

The scattered intensity at $q=0.0539 \AA^{-1}$ $(2 \theta=45.4 \mathrm{~min})$ was further analyzed on the basis of eq 10 in order to confirm further that the scattering arises from the fluctuations in the disordered state. It was found that the reciprocal intensity linearly decreases with increasing the reciprocal absolute temperature (Figure 4) except for the lowest temperature $\left(45^{\circ} \mathrm{C}\right)$. Thus except for the lowest temperature at which the agreement between the theoretical and the experimental profiles also becomes relatively poor, the scattering is confirmed to arise from the thermal fluctuations in the disordered state.

If the deviation from the linearity at the lowest temperarure in the $I^{-1}$ vs. $T^{-1}$ plot

Table I. $\chi$-Values on monomer units as a function of temperature

\begin{tabular}{cccccccc}
\hline $\begin{array}{c}\text { Temp/ } \\
{ }^{\circ} \mathrm{C}\end{array}$ & 45 & 60 & 80 & 100 & 110 & 120 & 140 \\
\hline$\chi$ & 0.106 & 0.102 & 0.095 & 0.085 & 0.080 & 0.071 & 0.064 \\
\hline
\end{tabular}




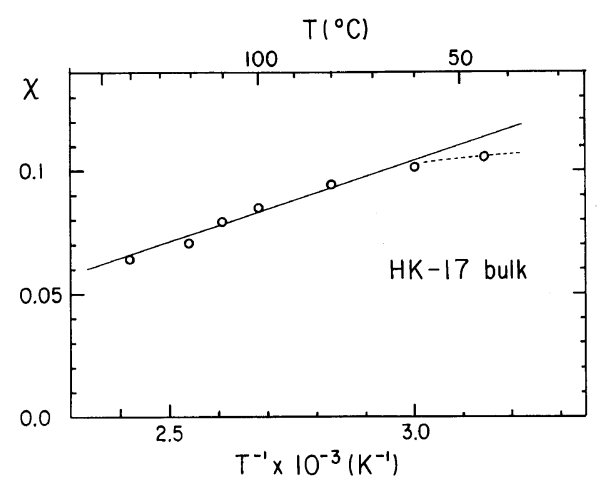

Figure 5. Temperature dependence of the interaction parameter $\chi$ on monomer units obtained by the best fits of the experimental and theoretical SAXS profiles for $\mathrm{HK}-17$ in bulk and in the disordered state.

should arise from the onset of the disorderorder transition (i.e., the microphase separation), it should be accompanied by the increase of $D$ at that temperature (see eq 7)..$^{5-8}$ However such tendency can be hardly seen. Consequently we tentatively conclude that the deviation is attributed to the glass transition of the block polymer in the disordered state. As the temperature is lowered the accidental thermal fluctuations increases and therefore the scattered intensity tends to increase because of increasing the $\chi$-parameter. If the temperature, however, is further lowered below the glass transition temperature, $T_{\mathrm{g}}$, the accidental thermal fluctuations themselves are frozen at $T_{\mathrm{g}}$ and therefore the scattered intensity would not increase any more but remains constant being equal to the intensity at $T_{\mathrm{g}}$ as shown by the broken horizontal line in Figure 4. If this is the case the apparent $\chi$-value estimated from the SAXS intensity will be also frozen at $T_{\mathrm{g}}$, i.e.,

$$
\chi=\chi\left(T_{\mathrm{g}}\right) \quad \text { at } \quad T<T_{\mathrm{g}}
$$

This effect is confirmed in Figure 5 and is drawn by the broken curve. In fact $T_{\mathrm{g}}$ of $\mathrm{HK}$ 17 in the disordered state estimated with Fox's equation $^{23}$ from the $T_{\mathrm{g}}{ }^{\text {' }} \mathrm{s}^{24}$ of PS $\left(107^{\circ} \mathrm{C}\right)$ and PI $\left(9^{\circ} \mathrm{C}\right)$ block chains in the ordered state is $51^{\circ} \mathrm{C}$ which is nicely in the temperature range where the deviation from the linearity in the
$I^{-1}$ vs. $T^{-1}$ plot becomes remarkable. We are now attempting to confirm this effect of the glass transition on the accidental thermal fluctuations by means of thermal analysis.

The $\chi$-values on monomer units for PS and $\mathrm{PI}$ in the thermal equilibrium regime are summarized by the equation,

$$
\chi=-0.0937+66 / T
$$

Our method to estimate $\chi$-values may be superior to the method which involves the determination from the phase diagrams of corresponding oligomers or mixtures based on random copolymers ${ }^{25,26}$ because in the latter method the determination of the binodal line or the cloud points for the bulk specimens is usually very difficult and the achievements of the thermal equilibrium are also very difficult and time-consuming. On the other hand in our method, the equilibrium can be easily achieved above $T_{\mathrm{g}}$. However, it should be noted that the $\chi$-values estimated by our method strongly depends on the accuracy of the theory. The $\chi$-values as given by eq 21 reasonably agree with the reported results. ${ }^{25,26}$

Acknowledgements. This work was supported in part by scientific grants from Asahi Glass Foundation for Industrial Technology, Japan and from Japan Securities Scholarship Foundation.

\section{REFERENCES}

1. K. Mori, A. Okawara, H. Hasegawa, and T. Hashimoto, Polym. Prepr. Jpn., 32, 1683 (1983).

2. K. Mori, H. Hasegawa, and T. Hashimoto, Polym. Prepr. Jpn., 33, 2267 (1984).

3. L. Leibler, Macromolecules, 13, 1602 (1980).

4. R. J. Roe, M. Fishkis, and C. J. Chang, Macromolecules, 14, 1091 (1981).

5. T. Hashimoto, M. Shibayama, and H. Kawai, Polym. Prepr., Am. Chem. Soc., Div. Polym. Chem., 23, 21 (1982).

6. T. Hashimoto, K. Kowsaka, M. Shibayama, and H. Kawai, Polym. Prepr., Am. Chem. Soc., Div. Polym. Chem., 24, 224 (1983).

7. T. Hashimoto, M. Shibayama, and H. Kawai, Macromolecules, 16, 1093 (1983). 
8. T. Hashimoto, Y. Tsukahara, and H. Kawai, Polym. J., 15, 699 (1983).

9. See for example, P. G. de Gennes, "Scaling Concepts in Polymer Physics," Cornell Univ. Press, Ithaca, New York, N.Y., 1979.

10. See for example, A. Ishihara, "Statistical Physics," Academic Press, New York, N.Y., 1971; H. Haken, "Synergestics. An Introduction, Nonequilibrium Phase Transitions and Self-Organization in Physics, Chemistry and Biology," Springer-Verlag, Berlin, 1978.

11. T. Hashimoto, M. Shibayama, and H. Kawai, Macromolecules, 13, 1237 (1980).

12. T. Hashimoto, M. Fujimura, and H. Kawai, Macromolecules, 13, 1660 (1980).

13. F. S. Bates, C. V. Berney, and R. E. Cohen, Macromolecules, 16, 1101 (1983).

14. D. J. Meier, Prepr. Polym. Colloq., Soc. Polym. Sci., Jpn., 83 (1977).

15. E. Helfand, Macromolecules, 8, 552 (1975).
16. R. L. Scott, J. Chem. Phys., 17, 268 (1949).

17. A. D. LeGrand and D. G. LeGrand, Macromolecules, 12, 450 (1979).

18. L. Leibler and H. Benoit, Polymer, 22, 195 (1981).

19. M. Benmouna and H. Benoit, J. Polym. Sci., Polym. Phys. Ed., 21, 1227 (1983).

20. Y. Tsukahara, N. Nakamura, T. Hashimoto, and H. Kawai, Polym. J., 12, 455 (1980).

21. T. Hashimoto, S. Suehiro, M. Shibayama, K. Saijo, and H. Kawai, Polym. J., 13, 501 (1981).

22. M. Fujimura, T. Hashimoto, and H. Kawai, Mem. Fac. Eng., Kyoto Univ., 43, 224 (1981).

23. T. G. Fox, Bull. Am. Phys. Soc., 1, 123 (1956).

24. K. Mori, H. Hasegawa, and T. Hashimoto, unpublished data in DSC study of block polymers.

25. N. A. Rounds and D. McIntyre, cited in E. Helfand and Z. R. Wasserman, Macromolecules, 9, 879 (1976).

26. R. J. Roe and W. C. Zin, Macromolecules, 13, 1221 (1980). 